\title{
Prospective Isolation of Cortical Interneuron Precursors from Mouse Embryonic Stem Cells
}

\author{
Asif Mirza Maroof, ${ }^{1,2,3}$ Keith Brown, ${ }^{1,2}$ Song-Hai Shi, ${ }^{2}$ Lorenz Studer, ${ }^{2}$ and Stewart A. Anderson ${ }^{3}$ \\ ${ }^{1}$ Program in Neuroscience, Weill Cornell Graduate School of Medical Sciences, ${ }^{2}$ Sloan Kettering Institute, and ${ }^{3}$ Department of Psychiatry, Weill Cornell \\ Medical College, New York, New York 10065
}

Despite their therapeutic potential, progress in generating fully differentiated forebrain neurons from embryonic stem cells (ESCs) has lagged behind that from more caudal regions of the neuraxis. GABAergic interneuron precursors have the remarkable ability to migrate extensively and survive after transplantation into postnatal cortex, making them an attractive candidate for use in cell-based therapy for seizures or other neuropsychiatric disorders. We have modified a mouse ESC line with an Lhx6-GFP reporter construct that allows for the isolation of newly generated cortical interneuron precursors. When transplanted into postnatal cortex, these cells can migrate into the cortical parenchyma, survive for months, and display morphological, neurochemical, and electrophysiological properties characteristic of mature interneurons. This work demonstrates that forebrain neuronal subtypes with complex traits can be generated from embryonic stem cells, and provides a novel approach to the study of cortical interneuron development and to the establishment of cell-based therapies for neurological disease.

\section{Introduction}

Embryonic stem cells (ESCs) are a powerful source for generating potentially unlimited numbers of specialized cells. Several strategies have been developed to direct ESCs toward neural fates, such as midbrain dopamine neurons, spinal motoneurons, GABAergic neurons, and cortical projection neurons (Lee et al., 2000; Wichterle et al., 2002; Eiraku et al., 2008; Gaspard et al., 2008; Tabar et al., 2008; Ideguchi et al., 2010). However, ESCbased approaches face a number of challenges that should be addressed for future application in biology and regenerative medicine. These include the fundamental question of whether a neuron generated from ESCs outside of the normal developmental milieu can acquire the complex neurochemical and physiological properties exhibited by the equivalent cell born in utero. In addition, a key technical challenge is to allow prospective isolation of specific neuronal types when the cells are committed to a given fate but retain sufficient plasticity for integration into the host.

Here we present data addressing these challenges by establishing a source of ESC-derived GABAergic interneurons. Cortical interneurons are a particularly interesting cell population due to their remarkable migratory capacity in postnatal cortex (Wichterle et al., 1999), their stereotypic differentiation after transplantation (Cobos et al., 2005; Alvarez-Dolado et al., 2006; Flames et al., 2007;

\footnotetext{
Received Aug. 28, 2009; revised Feb. 2, 2010; accepted Feb. 5, 2010.

This work was supported by a National Institute of Mental Health National Research Service Award (A.M.M.), the Starr Foundation (S.A., L.S., K.B., S.H.S.), NYSTEM (S.A., L.S.), and the National Institute of Mental Health (S.A.). We thank Jennifer Tyson, Darym Alden, and Faizzan Ahmad for technical support; and Mark Tomishima, Simon Butt, the Memorial Sloan-Kettering Cancer Center Flow Cytometry and Molecular Cytology core facilities, Vassilis Pachnis, Bernardo Rudy, Robert Hevner, and Eseng Lai for providing antibodies.

Correspondence should be addressed to Stewart A. Anderson, Weill Cornell Medical College, Box 244, 1300 York Avenue, New York, NY 10065. E-mail: saa2007@med.cornell.edu.

DOI:10.1523/JNEUROSCI.4255-09.2010

Copyright $\odot 2010$ the authors $\quad 0270-6474 / 10 / 304667-09 \$ 15.00 / 0$
}

Wonders et al., 2008), and their regulation of cortical activity. Abnormalities of cortical interneuron function have been implicated in a number of neuropsychiatric illnesses, including epilepsy, autism, and schizophrenia (Levitt et al., 2004).

Most cortical interneurons can be separated into three subgroups that express parvalbumin (PV), somatostatin (Sst), or calretinin $(\sim 30 \%$ of which colabel with Sst) (Kubota and Kawaguchi, 1994; Gonchar and Burkhalter, 1997; Xu et al., 2006). The PV- and Sst-expressing subgroups together account for $\sim 65 \%$ of cortical interneurons in mice (Tamamaki et al., 2003), and both subgroups originate primarily in the medial ganglionic eminence (MGE), where their fate determination requires the transcription factor Nkx2.1 (Xu et al., 2004, 2005; Butt et al., 2008). The LIM homeodomain factor Lhx6 is a direct target of Nkx2.1 and is critical to the specification of PV- and Sstexpressing phenotypic fates (Grigoriou et al., 1998; Liodis et al., 2007; Du et al., 2008; Zhao et al., 2008). Lhx6 is expressed in most MGE-derived interneurons from the time of cell cycle exit (Lavdas et al., 1999) through postnatal maturation (Cobos et al., 2005; Fogarty et al., 2007; Liodis et al., 2007).

Based on these developmental insights, we postulated that Lhx6 could serve as a useful marker to purify ESC-derived cortical interneuronal progenitors, and also to identify these cells following transplantation into mouse cortex. We used an Lhx6-GFP reporter construct to generate a transgenic mouse ESC (mES) line. This line permits the prospective isolation of GFP + cells that, following transplantation into postnatal cortex, migrate extensively and develop neurochemical and electrophysiological features characteristic of endogenous interneuron populations. The presence of highly specific differentiated properties in grafted Lhx6-GFP neurons in vivo suggests that ESC-based strategies are suitable for modeling developmental paradigms in the mammalian forebrain. 

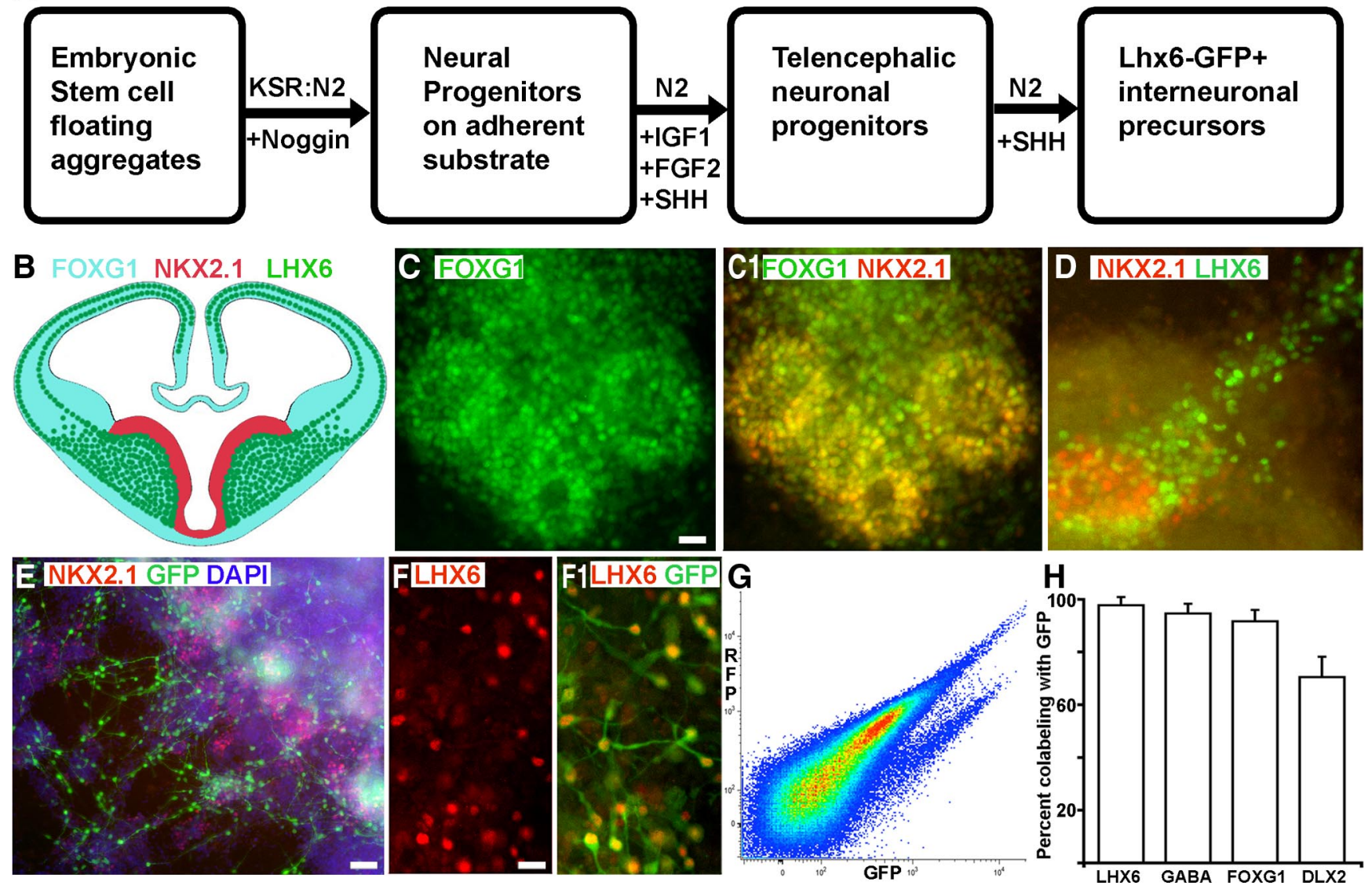

Figure 1. Generation of mouse ES cell-derived, MGE-like interneuronal precursors. $A$, The paradigm for differentiating ES cells toward ventral forebrain progenitors. $\boldsymbol{B}$, Model of the transcriptional profile of interneuronal precursors migrating in the developing telencephalon, as viewed through a coronal section at E13.5. FoxG1 is expressed throughout the telencephalon, while Nkx2.1 is expressed in the MGE and the preoptic area. Lhx6 is continuously expressed from the postmitotic state through adulthood. $C-E$, ES cells differentiated with the ventral telencephalon protocol for $12 \mathrm{~d}$. The sample in C shows the telencephalic marker FoxG1. Many of the FoxG1-expressing cells also express the MGE marker Nkx2.1 (yellow; ( 1). D, Lhx6+ cells appear to be streaming from an Nkx2.1 + cluster. $\boldsymbol{E}, \boldsymbol{F}$, Differentiated cells from the Lhx6::GFP reporter mES line. The GFP + cells appear to be streaming out of Nkx2.1+ progenitor domains $(\boldsymbol{E})$, and these cells colabel for Lhx6 protein $(\boldsymbol{F}, \boldsymbol{F 1}) . \mathbf{G}, \mathrm{FACS}$ analysis of the Lhx6::GFP line differentiated until day 12 shows a GFP + population clearly segregating from the autofluorescent background. Yield based on FACS (GFP + cells whose viability is established by DAPI exclusion) is $\sim 2 \%$. $\boldsymbol{H}$, GFP + cells were plated on glass coverslips and then immunolabeled for the indicated markers. The Lhx6::GFP + cells generally express the telencephalic marker FoxG1, the neurotransmitter GABA, Lhx6, and the ventral telencephalic marker Dlx2. There was $<1 \%$ colabeling for the dorsal telencephalic markers Pax6 or Tbr1. Scale bars: $(-E, 20 \mu \mathrm{m} ; \boldsymbol{F}, 10 \mu \mathrm{m}$.

\section{Materials and Methods}

Generation of the mES-Lhx6::GFP line. The previously generated Lhx6GFP bacterial artificial chromosome (BAC) from GENSAT (Gong et al., 2003) was modified for G418 selection as described previously (Tomishima et al., 2007). This BAC, which does not express an Lhx6 transcript, was then electroporated into a mouse ES line (J1, ATCC) to generate the Lhx6::GFP line. Karyotype and FISH analysis revealed a normal chromosomal arrangement and a single BAC integration site on chromosome 13 (data not shown).

ES cell culture. Mouse ES cells were grown on mouse embryonic fibroblasts in ES medium [knock-out DMEM (Invitrogen), 15\% FBS (Invitrogen-Thermo Fisher Scientific), L-glutamine, pen/strep, MEM nonessential amino acids, $\beta$-mercaptoethanol, and LIF (R\&D Systems)]. For differentiation, ES cells were treated with $0.05 \%$ trypsin and plated as single cells at 70,000 cells $/ \mathrm{ml}$ as described previously (Watanabe et al., 2005). Cells that became floating embryoid bodies were grown in a 1:1 mixture of KSR and N2 media supplemented with noggin $(250 \mathrm{ng} / \mathrm{ml}$, R\&D Systems). On differentiation day 5 (dd5), embryoid bodies (EBs) were mechanically dissociated using Accutase (Invitrogen) and plated onto polyornithine-, laminin-, and fibronectin-coated plates using highdensity droplets $(\sim 10,000$ cells/ $\mu \mathrm{l})$ in N2 medium with bFGF $(10 \mathrm{ng} / \mathrm{ml}$, day 5-8), IGF1 (20 ng/ml, day 5-8), and SHH (50 ng/ml, Shh-N-C25II, R\&D Systems). Medium was changed at dd 3 , dd5, dd 8 , and dd11, and cultures were typically fixed and analyzed by immunofluorescence or subjected to fluorescence-activated cell sorting (FACS) on dd 12 .

Cell sorting. At dd12, the cell cultures were treated with $0.05 \%$ trypsin for 5-8 min, followed by gentle trituration to dissociate to single cells. The dissociated cells were centrifuged at $1000 \mathrm{rpm}$ for $5 \mathrm{~min}$, resuspended in N2 + 20\% FBS + DAPI, and passed through a $40 \mu \mathrm{m}$ filter. The cell suspension was kept on ice until FACS Vantage sorting (BD Biosciences) and analyzed using Flojo software (Tree Star). Dead cells were excluded by gating for DAPI-negative events.

Cortical transplantation. Transplantation into the somatosensory neocortex of neonatal pups was conducted as described previously (Wonders et al., 2008). Twenty thousand to forty thousand cells were injected into the cortical plate at the following coordinates from bregma $(2.0 \mathrm{~mm}$ anterior, $2.5 \mathrm{~mm}$ lateral, $1.0 \mathrm{~mm}$ dorsal), targeting cortical layers 3-6. Care of animals was in accordance with institutional guidelines at the Weill Cornell Medical College.

Immunohistochemistry. Cells were fixed in $4 \%$ paraformaldehyde and were stained with the following primary antibodies: rabbit antiLhx6 (provided by Vassilis Pachnis, The National Institute for Medical Research, London, UK), -FoxG1 (provided by Eseng Lai, Merck, Whitehouse Station, NJ), -GABA (Sigma-Aldrich), -GFP (Invitrogen), -Dlx2 (Porteus et al., 1994), -Kv3.1 (provided by Bernardo Rudy, New York University School of Medicine, New York, NY), 

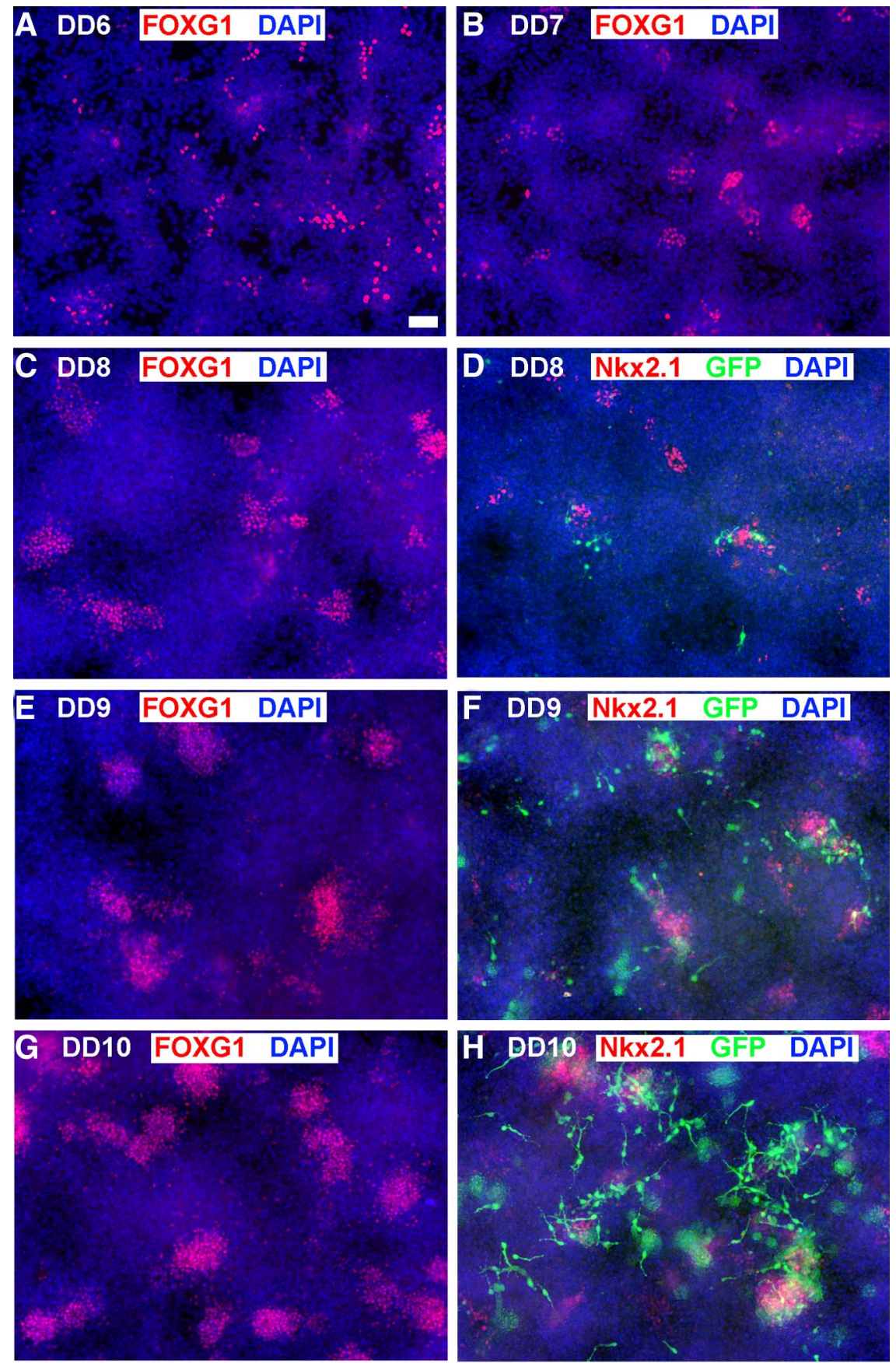

Figure 2. Development of FoxG1, Nkx2.1, and GFP in mouse ES Lhx6-GFP-derived cultures. Cells were differentiated by the protocol described in Figure $1 A$, in which floating embryoid bodies are dissociated and plated on adherent substrate at dd5. Blue signal shows the nuclear staining for DAPI in all panels. $A-C, E, G$, The telencephalic marker FoxG1 begins to be expressed in scattered cells by $1 \mathrm{~d}$ after plating (dd6), and clusters of these cells increase in size and number through dd10. D, F, H, Nkx2.1 (red signal), expressed in a substantial subset of FoxG1 + cells in these cultures (Fig. 1C1), is expressed in a few scattered cells at day 7 (D) and increases similarly to FoxG1 expression through day $10(\boldsymbol{H})$. Lhx6-GFP expression (green signal) is apparent in differentiating-appearing, process-bearing cells that often occur adjacent to Nkx2.1 + clusters. Lhx6-GFP expression is rare at dd8 (D), and increases substantially by dd $10(\boldsymbol{H})$. Scale bar, $100 \mu \mathrm{m}$.

-Tbr1 (provided by Robert Hevner Center for Integrative Brain Research, Seattle, WA), -neuropeptide Y (NPY) (Immunostar), -calretinin (Swant), and -Ki67 (Neomarkers); mouse anti-Nkx2.1 (Neomarkers) and -parvalbumin (Swant); rat anti-somatostatin (Millipore Bioscience Research Reagents); and chicken anti-GFP (Abcam). Secondary antibodies were conjugated to Alexa fluorophores (Invitrogen) or Cy5 (Jackson ImmunoResearch).
Quantification of transplant distribution. To identify the relative distribution of transplanted cells, brains were sectioned in the coronal plane at $50 \mu \mathrm{m}$ on a vibrating microtome (Leica). Every fifth section was labeled with rabbit-anti GFP immunofluorescence and the nuclear stain DAPI. Profile counts of GFP+ cells in the cortex, essentially all of which had neuronal morphologies after the $1 \mathrm{~d}$ posttransplant (DPT) time point, were conducted. As no cell core remained at the injection site at 7 DPT or 30 DPT, the section with the highest number of cell profiles was taken as the presumptive injection region, and distributions were calculated in $250 \mu \mathrm{m}$ bins extending in the rostral-caudal axis from this region. The number of cell profiles per bin was estimated by multiplying the number of profiles in the given section by 5 . Since this section was located at the distal end of each bin relative to the apparent injection region, the extrapolated counts per bin would be expected to underestimate total cell numbers since there is a clear decline in cells with distance from the injected region (see Fig. 2). However, as profile counts were used, and this method overcounts cells per section, the underestimation would not be substantial. This method provides an efficient way to quantify relative distributions of cells across transplanted brains and ages.

Electrophysiological recording. Electrophysiology was performed on mice [postnatal day 16 (P16)-P29] that had undergone transplantation as above. Animals were anesthetized and decapitated. The brain was then quickly removed and transferred to ice-cold sucrose cutting solution containing the following: $214 \mathrm{~mm}$ sucrose, $2.5 \mathrm{~mm} \mathrm{KCl}, 24 \mathrm{~mm} \mathrm{NaHCO}_{3}, 1.25 \mathrm{~mm}$ $\mathrm{NaH}_{2} \mathrm{PO}_{4}, 11 \mathrm{~mm}$ D-glucose, $10 \mathrm{~mm} \mathrm{MgCl}_{2}$, and $0.5 \mathrm{~mm} \mathrm{CaCl}_{2}$ (osmolarity $\sim 315 \mathrm{mOsm}$ ), bubbled with $5 \% \mathrm{CO}_{2} / 95 \% \mathrm{O}_{2}$. Coronal cortical slices (200-300 $\mu \mathrm{m}$ ) were prepared using a vibratome (VT1000S; Leica Microsystems). Slices recovered in artificial CSF (ACSF) containing the following: $119 \mathrm{~mm} \mathrm{NaCl}, 2.5 \mathrm{~mm}$ $\mathrm{KCl}, 26.2 \mathrm{~mm} \mathrm{NaHCO}_{3}, 2.5 \mathrm{~mm} \mathrm{CaCl}_{2}, 1.3 \mathrm{~mm}$ $\mathrm{MgCl}_{2}, 1 \mathrm{~mm} \mathrm{NaH} \mathrm{PO}_{4}$, and $20 \mathrm{~mm}$ glucose ( $\mathrm{pH} 7.4$, osmolarity $\sim 300 \mathrm{mOsm}$ ), bubbled with $5 \% \mathrm{CO}_{2} / 95 \% \mathrm{O}_{2}$ at room temperature for a minimum of $1 \mathrm{~h}$ before recording. Slices were constantly perfused with ACSF during recordings. Whole-cell recordings were made from GFP-positive neurons located in layers II-V of the neocortex. GFP-positive cells were typically 40-70 $\mu \mathrm{m}$ below the surface of the slice. Patch electrodes (5-8 M $\Omega$ ) were filled with intracellular solution containing the following: 130 mM K-gluconate, $16 \mathrm{~mm} \mathrm{KCl,} 2 \mathrm{~mm} \mathrm{MgCl}_{2}, 10$ mM HEPES, 0.2 mм EGTA, $4 \mathrm{~mm} \mathrm{Na}_{2}$-ATP, and $0.4 \mathrm{mM} \mathrm{Na}_{3}$-GTP (pH 7.25, osmolarity $\sim 290 \mathrm{mOsm}$ ). The membrane potential of each cell was identified shortly after rupturing the patch and periodically during the course of the experiment to ensure there was no significant deterioration of the health of the cell. Spontaneous miniature synaptic currents were recorded in voltage-clamp mode held at $-60 \mathrm{mV}$. Depolarizing and hyperpolarizing current steps $(0.2 \mathrm{~Hz}$; duration $500 \mathrm{~ms})$ were applied to the cells to help characterize their electrophysiological profile. Cells that showed significant rundown were discarded. All parameters were mea- 

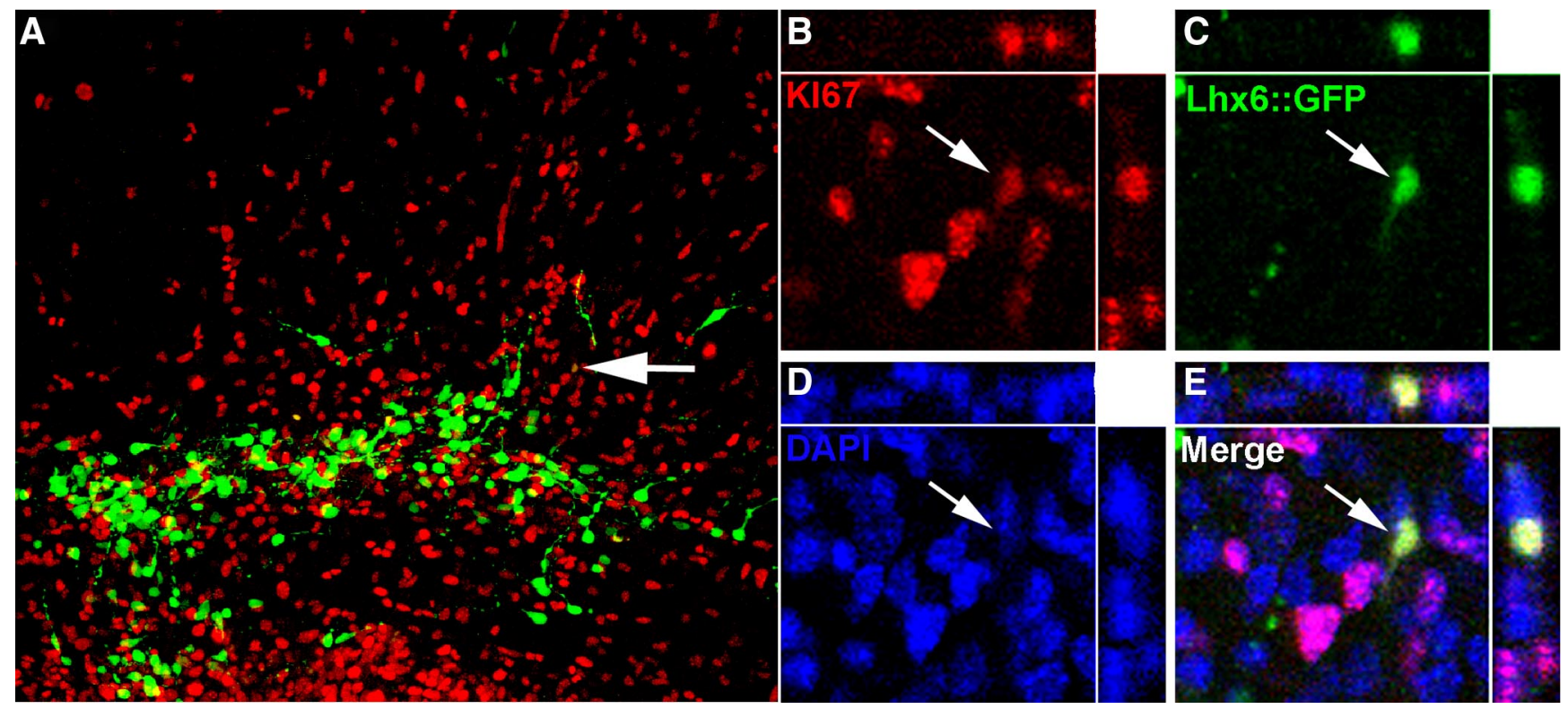

Figure 3. Nearly all Lhx6::GFP + cells are postmitotic $1 \mathrm{~d}$ after transplantation. $A$, Immunofluorescence labeling for GFP in a $50 \mu \mathrm{m}$ coronal section showing the location of an injection site $1 \mathrm{~d}$ following transplantation into neonatal cortex. $\boldsymbol{B}-\boldsymbol{E}$, Same view of the cell indicated by the arrow in $\boldsymbol{A}$ showing Ki67 (B), GFP ( $\boldsymbol{C}$ ), and DAPI ( $\boldsymbol{D}$; merged image in $\boldsymbol{E})$. Colabeling of GFP and the cell cycle marker ki67 is very rare $(<1 \%)$, indicating that Lhx6::GFP + cells have generally exited the cell cycle either before or shortly after the time of transplantation. No evidence of tumor formation was found in any of the over two dozen transplantations that have been evaluated at $1-240 \mathrm{~d}$ following transplantation.
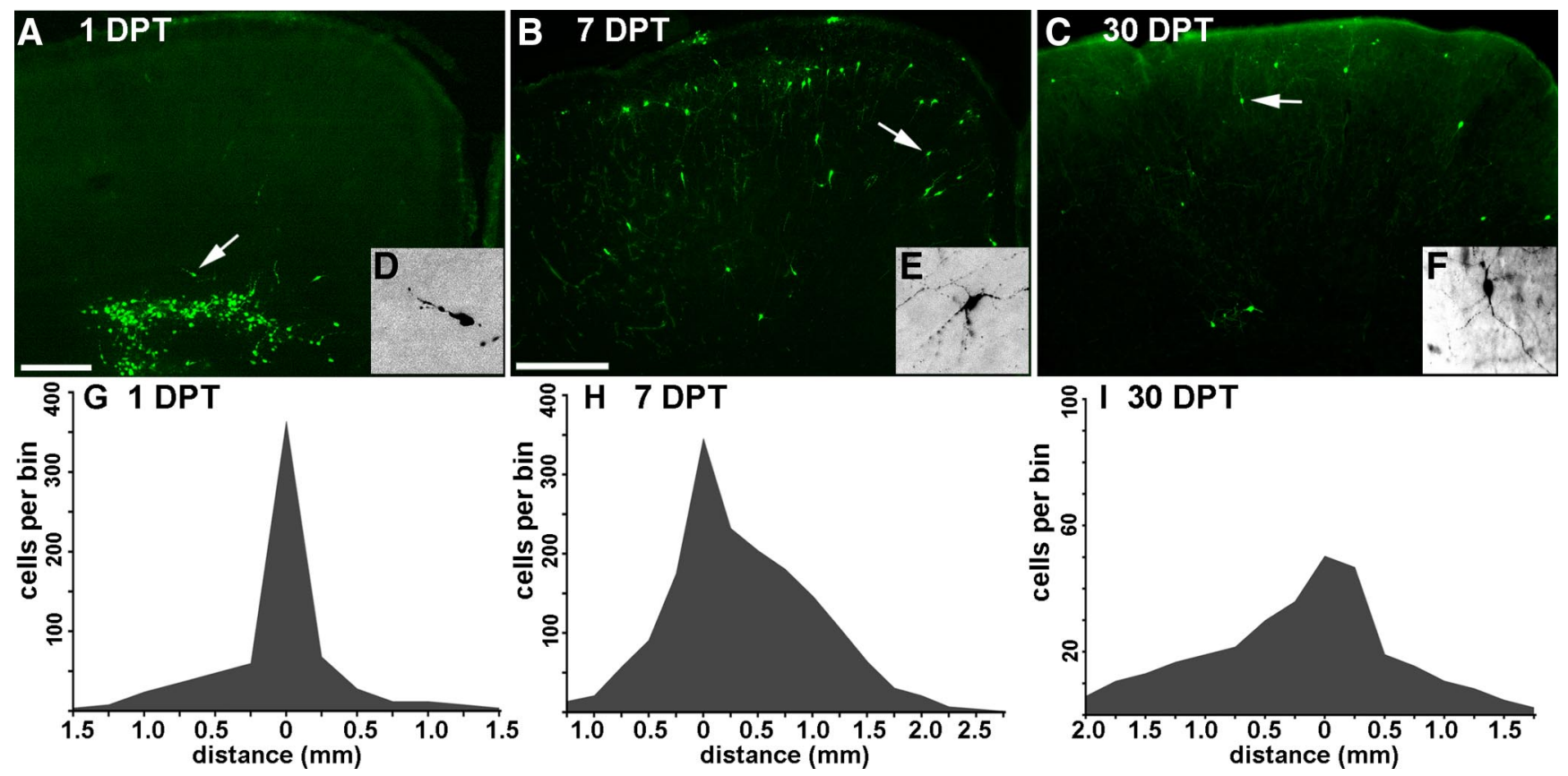

Figure 4. Migration and survival of Lhx6::GFP + cells transplanted into neonatal cortex. A-F, Immunofluorescence labeling of GFP in $50 \mu \mathrm{m}$ coronal sections. Arrows indicate cells shown at higher magnification in the insets ( $\boldsymbol{A}$ and $\boldsymbol{D}$ show the same section shown in Fig. 3). At 1 DPT, the GFP + cells were distributed close to the injection site, and many appear to be migrating into the cortical parenchyma. $\boldsymbol{B}, \boldsymbol{E}, \mathrm{At} 7 \mathrm{DPT}$, the GFP + cells are much more broadly distributed, and many have multipolar morphologies suggestive of postmigratory neurons. $\boldsymbol{C}, \boldsymbol{F}$, The mediolateral extent of these cells at 30 DPT is $2.5 \pm 0.3 \mathrm{~mm}$. $\mathbf{G}-\mathbf{I}$, Distributions of transplanted cells $1(\mathbf{G}, n=3), 7(\boldsymbol{H}, n=4)$, or $30(\boldsymbol{I}, n=5)$ days after transplantation. The $x$-axes show rostral- caudal distance from the injection site. The $y$-axes show cell profile number per $250 \mu \mathrm{m}$ bin made conservatively by multiplying the number of GFP + cell profiles in the most distal section of that bin by 5 . In $I$, the $y$-axis extends to 100 cells. G, After 1 DPT, the bulk of transplanted cells are within $300 \mu \mathrm{m}$ of the injection site. $\boldsymbol{H}$, By 7 DPT, some of the cells have dispersed as far as $2 \mathrm{~mm}$ from the injection site. I, Although the survival or detectability of GFP + cells had significantly decreased by $30 \mathrm{DPT}$, the rostral-caudal distribution is approximately equivalent to that seen at 7 DPT. Scale bars: $\boldsymbol{A}, 200 \mu \mathrm{m} ; \boldsymbol{B}, \boldsymbol{C}, 400 \mu \mathrm{m}$.

sured for a minimum of three trials for each cell, and the average value was calculated. Spike threshold for each cell was identified with incremental $10 \mathrm{pA}$ steps until action potentials were triggered. Firing characteristics of individual cells were measured between threshold and maximal firing rate until failure. To test whether injection of hyperpo- larizing current resulted in rebound spike activity, neurons were stepped between -40 and $-100 \mathrm{mV}$.

Cells were classified according to the criteria established by Fishell and colleagues (Butt et al., 2005; Miyoshi et al., 2007) and the Petilla conference (Ascoli et al., 2008). Briefly, cells that fire at $>50 \mathrm{~Hz}$, exhibit a 


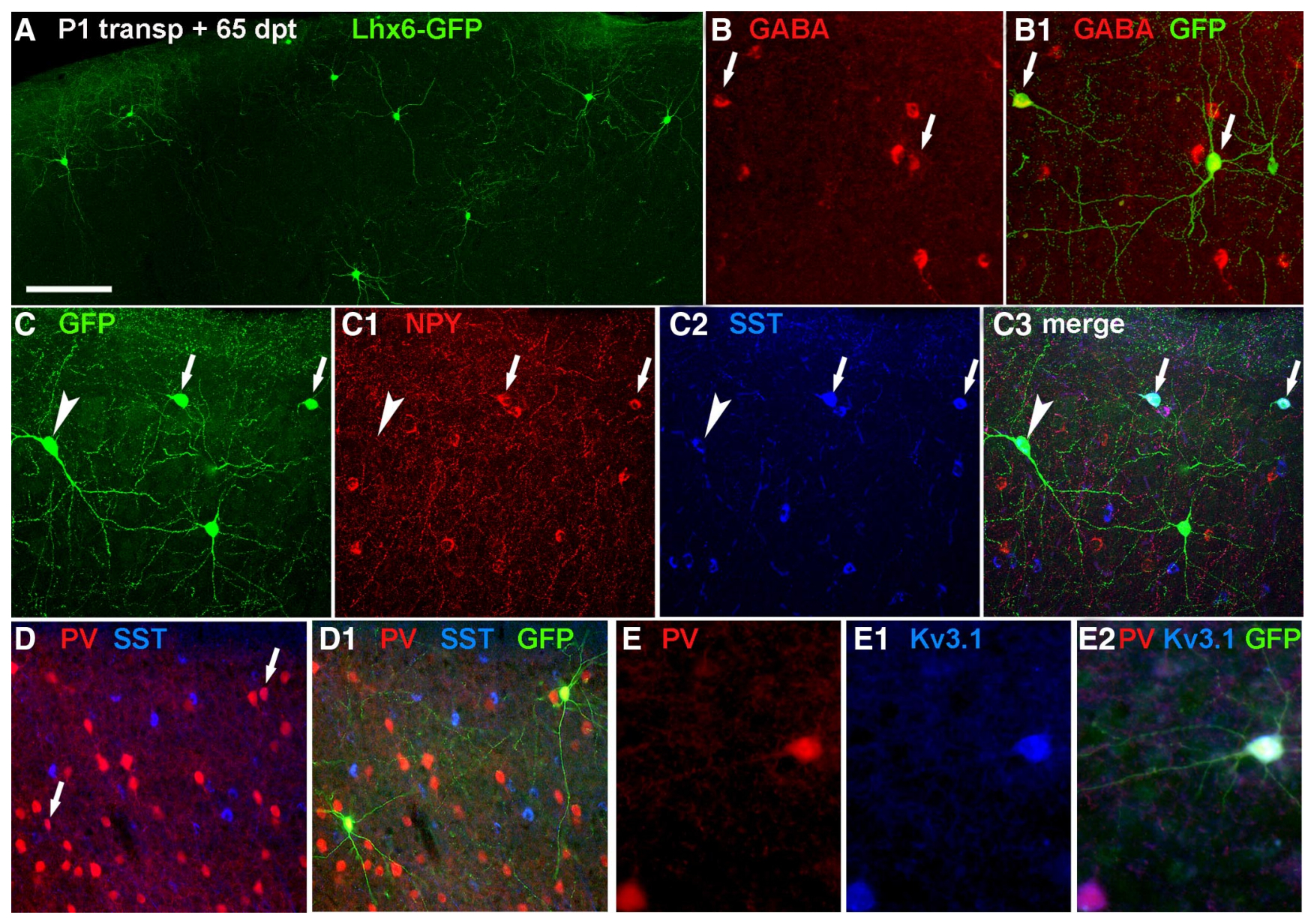

Figure 5. Transplantation of mES-derived, Lhx6::GFP + cells into postnatal cortex gives rise to interneuron-like cells. Lhx6::GFP + cells were differentiated, collected by FACS, then transplanted into the cortical plate of neonatal pups. Shown is immunolabeling of $50 \mu \mathrm{m}$ coronal sections taken at 65 DPT. $A$, The GFP + cells mainly exhibit multipolar, aspiny morphologies commonly present in cortical interneurons. Scale bar, $100 \mu \mathrm{m} . \boldsymbol{B}, \boldsymbol{B} 1$, The majority of these cells ( $>85 \%$, see Results) express GABA. C $-\mathbf{C}$, Confocal images of a section labeled for GFP (C), NPY (C1), and somatostatin (SST; C2; merged image in C3). As occurs ex vivo, there is partial overlap between the NPY + and SST + interneuron subgroups (arrowheads show SST-expressing ES-derived cells, small arrows indicate cells that coexpress NPY). D, D1, Colabeling of two GFP + cells with PV (arrows in D show colabeled cells in D1). As expected, the Lhx6::GFP and PV + colabeled cells do not express SST (blue pseudocolor from Cy5 signal), but express the potassium channel KV3.1 (E-E2).

consistent interspike interval with little to no adaptation, exhibit a deep, fast afterhyperpolarization (AHP), and exhibit no rebound spike activity are categorized as fast spiking (FS). Cells that were non-FS (NFS) were categorized into three groups: stuttering, adapting or accommodating (NFS1), or nonadapting (NFS2). Both NFS1 and NFS2 cells have small biphasic AHPs and a pronounced delay to spike at threshold, but most of the NFS1 cells exhibit considerable adaptation in spike frequency, whereas NFS2 cells often do not (Miyoshi et al., 2007).

For postrecording immunocytochemistry, $0.5 \%$ Neurobiotin (Vector Labs) was included in the internal solution. After recording, slices were fixed in $4 \%$ PFA for $4 \mathrm{~h}$ and proceeded to perform immunohistochemical analysis for GFP (Abcam chicken anti-GFP, Alexa goat anti-chicken 488), Neurobiotin (Jackson ImmunoResearch streptavidin-conjugated 546), and either PV (mouse anti-PV, Millipore Bioscience Research Reagents; donkey anti-mouse Cy5, Jackson ImmunoResearch) for fastspiking cells (7 of 19 recorded) or somatostatin (rat anti-Sst, Millipore Bioscience Research Reagents; donkey anti-rat Cy5, Jackson ImmunoResearch) for the non-FS cells (12 of 19).

\section{Results}

Generation of mouse ES cell-derived interneuronal precursors

Previous work has demonstrated that ESCs can be directed toward telencephalic progenitors, including those that differentiate into neurons expressing the generally inhibitory neurotransmitter GABA (Watanabe et al., 2005; Gaspard et al., 2008). Since FoxG1 + and Nkx2.1+ progenitors give rise both to GABAergic interneurons and projection neurons (Fogarty et al., 2007; Xu et al., 2008), we sought to determine whether cortical or striatal GABAergic interneurons can be generated from mouse ES cells. Using a modification of a protocol for the generation of ventral telencephalic cells (Fig. 1 A,B) (Watanabe et al., 2005), we found that $\mathrm{mES}$ cells can be directed to differentiate and aggregate into rosette-like clusters that strongly express both FoxG1 and Nkx2.1 (Fig. 1C,C1). By dd12, streams of cells appeared to emanate from these Nkx2.1+ clusters, and they expressed Lhx6 (Fig. 1D), a marker of most MGE-derived interneurons that is expressed from shortly before cell cycle exit through postnatal maturation (Lavdas et al., 1999; Liodis et al., 2007; Du et al., 2008).

Using our established protocol (Tomishima et al., 2007), we next generated ESC lines containing an Lhx6-GFP bacterial artificial chromosome that produces accurate reporter expression in Lhx6-expressing cells in vivo (Gong et al., 2003). Several GFPexpressing lines were generated and confirmed to express LHX6 protein in GFP+ cells (Fig. 1F,F1), and one of these lines, confirmed to have a normal karyotype, was chosen for detailed char- 
acterization. While our high-density droplet method of plating the embryoid bodies on day 5 consistently produced GFP + cells, we also observed GFP + cells in the "en bloc" method described by Watanabe et al. (2005), and when the cells were plated in a high-density dissociated monolayer (supplemental Fig. S1, available at www.jneurosci.org as supplemental material). No FoxG1, Nkx2.1, or GFP expression was detected at day 12 when embryoid bodies were plated as single cells at clonal density (data not shown), precluding analysis of whether rosettelike clusters of Nkx2.1+ and FoxG1+ cells derive from single progenitors. Although all three successful plating protocols produced Lhx6-GFP+ cells that colabel with GABA, most GABAexpressing cells in these cultures did not express Lhx6-GFP (supplemental Fig. S1, available at www.jneurosci.org as supplemental material). Since nearly all of the cells showing immunofluorescent signal for LHX6 were also GFP-expressing (Fig. $1 F$ ), this result is consistent with the notion that this protocol, like the forebrain itself, generates a diverse group of neuronal precursors.

Lhx6-GFP + cells were isolated by FACS, and the GFP + cells, which were $\sim 2 \%$ of the total viable cells (Fig. $1 G$ ), were characterized after acute plating (Fig. $1 H$ ). These cells expressed the general telencephalic marker FoxG1 $(91.6 \pm 4.4 \%)$, the subpallial marker Dlx2 (70.5 \pm 7.7\%), Lhx6 itself $(97.7 \pm 3.1 \%)$, and GABA $(94.6 \pm 3.7 \%)$ (Fig. $1 H$ ). Very rarely $(<1 \%)$ did Lhx6-GFP+ cells express the pallial progenitor marker Pax6 or Tbr1, a marker of most postmitotic neurons derived from the cerebral cortical proliferative zone (data not shown).

Using the protocol described in Figure $1 A$, we sought to determine the temporal expression of ventral transcription factors FoxG1, Nkx2.1, and Lhx6-GFP. After dissociating the embryoid bodies and plating the cells onto an attachable substrate in high-density droplets (see Materials and Methods), a few FoxG1+ cells appeared scattered throughout the culture on $1 \mathrm{~d}$ after plating (dd6). By dd8 rosette-like clusters began to form (Fig. $2 A-C)$. Nkx2.1+ cells, expressed by a subset of those that express FoxG1 (Fig. 1C1), are delayed relative to FoxG1 by $\sim 1$ $\mathrm{d}$ but follow a similar pattern through dd10 (Fig. $2 D, F, H$ ). GFP is apparent in a few cells at dd7, and also expands considerably through dd10 (Fig. 2D,F,H). This progression of FoxG1, Nkx2.1, and Lhx6 expression parallels events occurring in
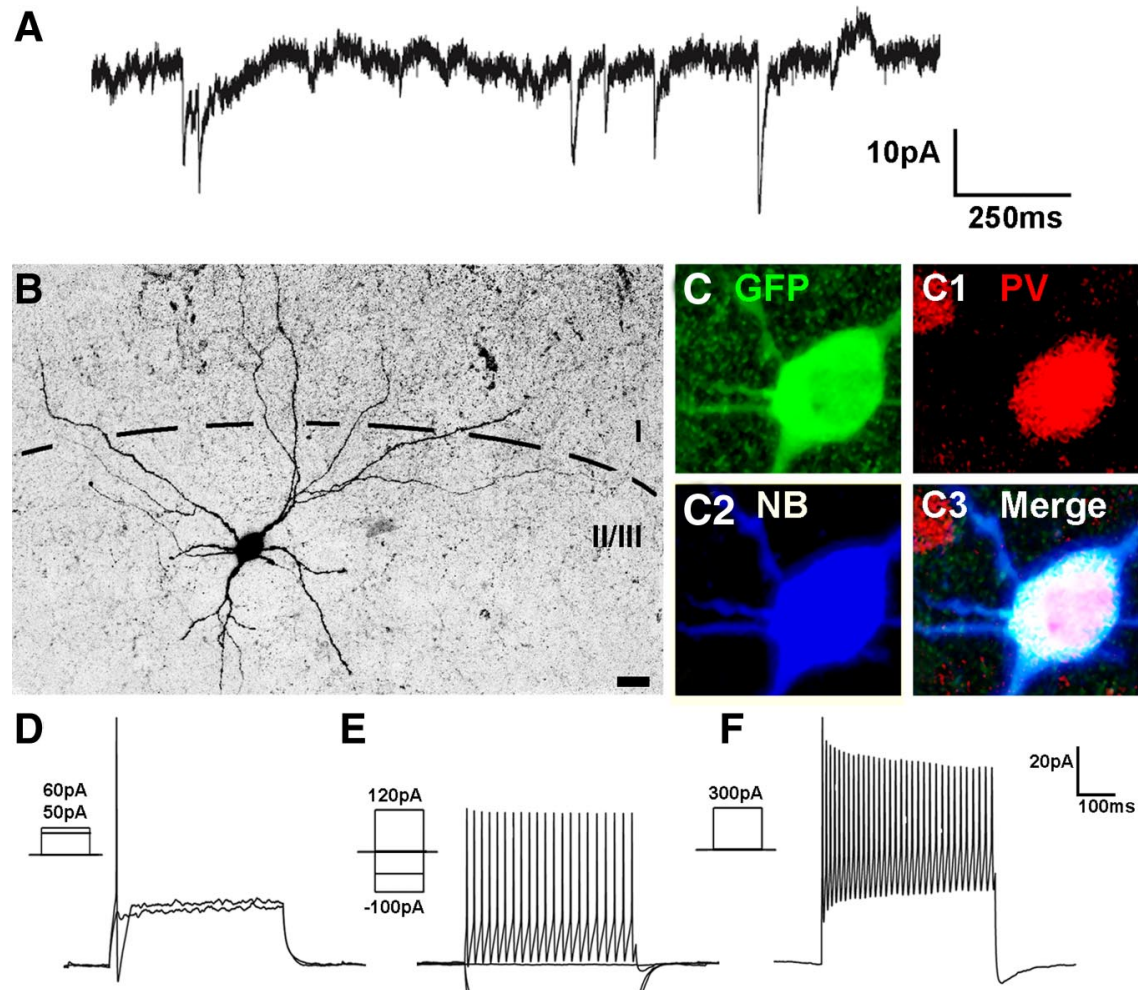

E
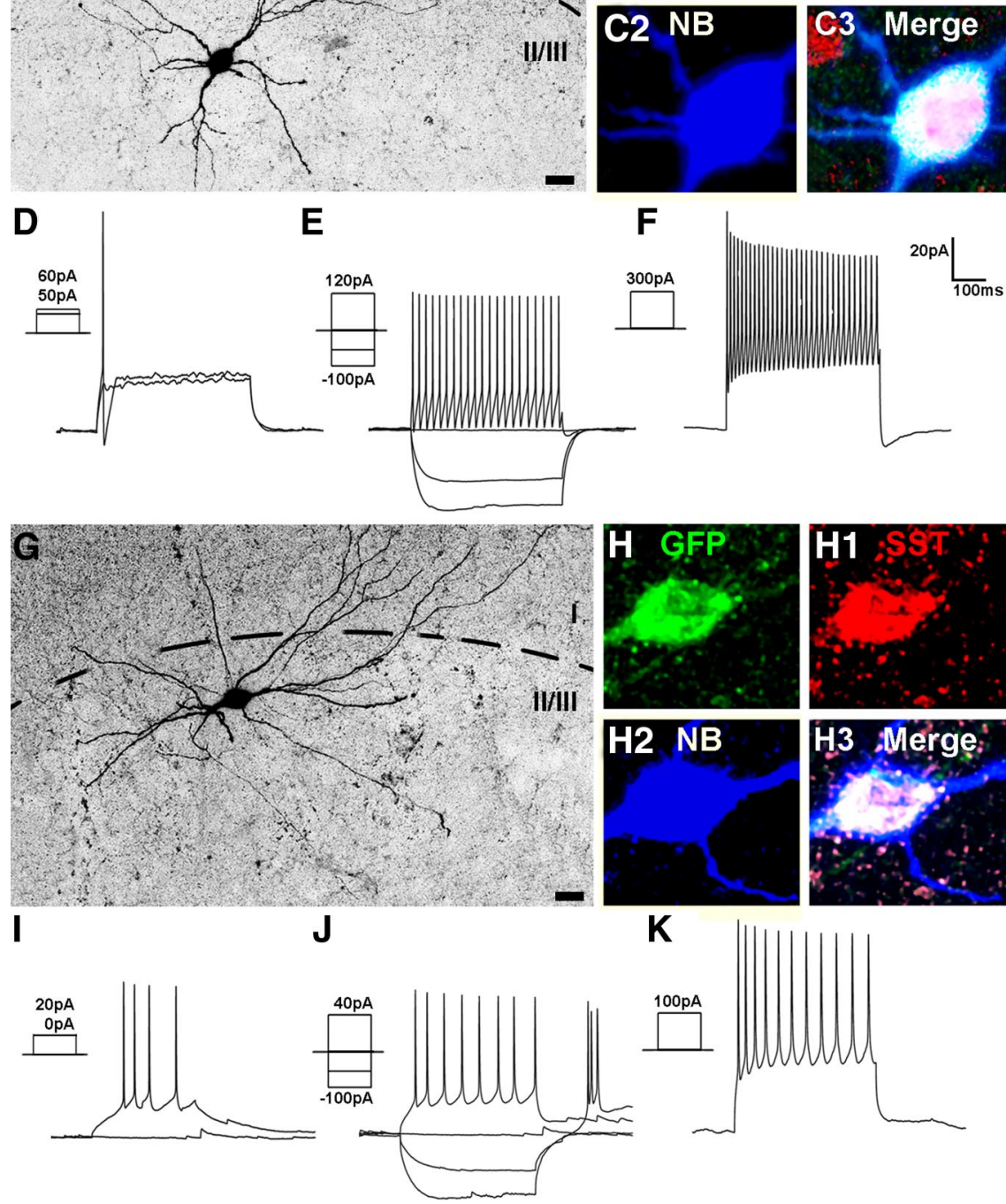

Figure 6. mES-derived, Lhx6-GFP + cells exhibit physiological and neurochemical characteristics of cortical interneurons. Lhx6-GFP cells were differentiated and transplanted as in Figure 5. Twenty-two days after transplantation, slices from these animals were subjected to whole-cell patch-clamp recordings and fluorescence labeling. $A$, Spontaneous synaptic inputs recorded from a GFP + cell voltage-clamped at $-60 \mathrm{mV}$. $\boldsymbol{B}-\boldsymbol{K}$, Neurochemical profiles and physiological responses of two transplanted cells. Images are formed from collapsed stacks taken by confocal microscopy. $\boldsymbol{B}, \boldsymbol{G}$, Monochrome images of the Neurobiotinstreptavidin-Alexa 546 signals. Higher-magnification views are shown in $\mathbf{C}-\mathbf{C}$ and $\boldsymbol{H}-\boldsymbol{H} \mathbf{3}$, where the Neurobiotin (NB) signal ( $\mathbf{C}$, H2) has been pseudocolored blue to demonstrate that the same cell soma is being imaged as that shown at lower magnification. These cells were subjected to electrophysiological recordings, with current injection protocols testing threshold current injection $(\boldsymbol{D}, I)$, response to hyperpolarizing current injection and $2 \times$ threshold $(\boldsymbol{E}, \boldsymbol{J})$, and suprathreshold $(5 \times)$ current injection that elicited discharge of action potentials at a near-maximal firing frequency $(\boldsymbol{F}, \boldsymbol{K})$. The cell in $\boldsymbol{B}$ colabels for GFP $(\boldsymbol{C})$, PV $(\boldsymbol{C} \mathbf{1}$; red pseudocolored from (y5 signal), and NB (C2) and exhibited a fast-spiking discharge pattern $(\boldsymbol{E}, \boldsymbol{F})$ typical of PV-expressing interneurons. The cell in $\mathbf{G}$ colabels for GFP $(\boldsymbol{H})$, somatostatin (SST, $\boldsymbol{H}$; ; red pseudocolored from Cy5 signal), and NB (H2) and exhibited a rebound, adapting, non-fast-spiking pattern $(\boldsymbol{I}-\boldsymbol{K})$ typical of many SST-expressing interneurons. Scale bars: $\boldsymbol{B}, \mathbf{G}, 20 \mu \mathrm{m}$. 
the ventral telencephalon from embryonic day 9 to embryonic day 12 .

\section{Migration and survival of Lhx6::GFP cells}

To characterize the fate potential of the Lhx6::GFP line, cells were differentiated for $12 \mathrm{~d}$ (Fig. $1 A$ ), collected by FACS for GFP expression (Fig. 1G), and transplanted into the cortical plate of neonatal mice. MGE-derived progenitors transplanted into both neonatal and adult mouse cortex have been shown to survive, migrate, and differentiate into several subgroups of cortical interneurons (Wichterle et al., 1999; Cobos et al., 2005; AlvarezDolado et al., 2006; Fogarty et al., 2007; Wonders et al., 2008). Relative to progenitors of the cortex or the lateral ganglionic eminence (LGE), this robust migration was found to be unique to MGE-derived progenitors (Wichterle et al., 1999, 2003; Anderson et al., 2001).

One day after transplantation into layers $5-6$ of the P 1 cortex, $\mathrm{GFP}+$ cells were distributed close to the injection site, with many cells exhibiting a bipolar morphology typical of migrating neurons (Figs. 3A, 4A). Consistent with previous findings that Lhx6 begins to be expressed around the time of cell cycle exit (Lavdas et al., 1999; Du et al., 2008), there was minimal colabeling of GFP and Ki67 at 1 DPT (Fig. 3). Indeed, no tumors were found in any of the transplants analyzed at any age in this study.

By 7 DPT, GFP + cells were distributed throughout cortical layers 2-6 and dispersed over a 2-mm-wide range along both the rostral-caudal and the medial-lateral axes, with some cells reaching a distance of $3 \mathrm{~mm}$ from the injection site (Fig. $4 \mathrm{~B}$ ). In contrast to 1 DPT, by 7 DPT most of these cells assumed multipolar morphologies typical of differentiating, postmigratory interneurons (Fig. 4E). At 30 DPT (Fig. 4C), cells exhibiting more complex neuritic arbors (Fig. $3 F$ ) were distributed over a similar range as at $7 \mathrm{DPT}$, but smaller total numbers of GFP + cells were observed. Quantification of the distribution of GFP + cells along the rostral-caudal axis from the injection site at 1, 7, and 30 DPT is presented in Figure $4 G-I$. These data suggest that migration is completed after 1 week post-transplantation, and that Lhx6::GFP cells undergo migratory dispersion from the transplantation site in a manner that is similar to MGE-derived transplants, and dissimilar to those from LGE or cortex. Although there was a dramatic decline in the detection of GFP + cells at 30 DPT (1-2\% of grafted cells vs $10 \%$ of grafted cells at 7 DPT), the GFP+ population at 30 DPT appeared relatively stable, as similar cell numbers and distributions were observed at 65 DPT (Fig. 5A). Transplanted GFP + neurons remain detectable for at least 240 DPT (supplemental Fig. S2, available at www.jneurosci.org as supplemental material).

\section{Transplanted Lhx6::GFP cells exhibit cortical interneuron-like neurochemical properties}

To determine whether ESC-derived cells acquire neurochemical characteristics of cortical interneurons, transplanted Lhx6:: $\mathrm{GFP}+$ cells were examined by immunofluorescence for interneuron markers at 65 DPT (Fig. 5). Like MGE-derived cortical interneurons, most GFP+ cells express GABA (Fig. 5B,B1) (161/189 from 5 transplantations; $85.7 \pm 3.5 \%$ ), as well as the subgroupdefining markers PV or Sst (Fig. 5C-D1). Typical of native Sstexpressing interneurons, GFP+ neurons that colabeled for Sst did not express PV or KV3.1, but did frequently colabel with NPY (Fig. 5C-C3) (present in 57 of 179 cells colabeled for Sst and GFP + cells). Typical of native PV + interneurons, nearly all $\mathrm{GFP}+$ neurons that colabeled for PV also expressed the potassium channel KV3.1 (Fig. 5E-E2) (38/39 cells). Counts of cells in
Table 1. Electrophysiological characteristics of Lhx6::GFP + cells after transplantation into neonatal cortex, based on whole-cell patch-clamp recordings

\begin{tabular}{llcc}
\hline Age & Interneuron type & No. cells & Avg. RMP $(\mathrm{mV} ; \pm$ SD $)$ \\
\hline 14 & Immature & 3 & $-44 \pm 6$ \\
$20-26$ & Immature & 7 & $-44 \pm 4.4$ \\
$20-26$ & NFS2 & 11 & $-57.2 \pm 6.8$ \\
$20-26$ & NFS1 & 7 & $-59.2 \pm 9.9$ \\
$20-26$ & C-STUT & 6 & $-60.2 \pm 6.3$ \\
$20-26$ & FS & 11 & $-63.5 \pm 6$ \\
\hline
\end{tabular}

C-STUT, Stuttering. See Materials and Methods and Miyoshi et al. (2007).

triple-labeled (PV-Sst-GFP) sections from four different transplanted animals revealed that out of $290 \mathrm{GFP}+$ cells, 185 (64\%) coexpressed Sst, and 30 (10\%) express PV. In sum, Lhx6::GFP ES cells can be differentiated into neurons that express appropriate combinations of proteins known to be present in Lhx6+ interneuron subpopulations in the mouse cerebral cortex.

\section{Transplanted Lhx6::GFP+ cells have MGE-interneuron-like spiking characteristics}

The data on cell migration and neurochemical differentiation strongly suggest that Lhx6::GFP + cells differentiate into functional neurons that exhibit features of MGE-derived cortical interneuron subgroups in vivo. To test this directly, we performed electrophysiological studies to examine the biophysical properties and the functional incorporation of transplanted Lhx6::GFP+ cells in situ. Under the visual guidance of fluorescence illumination, whole-cell patch-clamp recordings were performed on GFP + cells in acutely prepared cortical slices between 14 and 26 DPT (Fig. 6). We recorded a total of 45 GFP + cells (Table 1) with an average resting membrane potential (RMP) of $-54.7 \pm 6.5$ $\mathrm{mV}$. As expected, some cells recorded at early time points exhibited immature firing characteristics $(n=10$, including all 3 cells from 14 DPT and 7 cells between 20 and 26 DPT), such as a less hyperpolarized resting membrane potential (average RMP = $-43.6 \pm 5.2 \mathrm{mV}$ ) and a higher threshold for firing action potentials (data not shown). In contrast, cells recorded at later time points exhibited more hyperpolarized resting membrane potentials (average RMP $=-60 \pm 7.2 \mathrm{mV}$ ) and firing properties characteristic of cortical interneurons (Fig. $6 D-F, I-K$, Table 1). These included FS and three classes of NFS patterns, stuttering nonpyramidal, NFS1 (accommodating), and NFS2 (nonaccommodating) (Kawaguchi and Kubota, 1996; Markram et al., 2004; Butt et al., 2005). NFS patterns (see Materials and Methods) frequently correlate with Sst expression, while cells exhibiting fast-spiking firing patterns are typical of the PV-expressing interneuron subgroup (Kawaguchi and Kubota, 1996). Three of seven fast-spiking neurons (maximal firing rate $>50 \mathrm{~Hz}$ at room temperature) expressed detectable levels of PV (Fig. 6C), while 8/12 non-FS neurons expressed Sst. Finally, under voltage-clamp conditions, GFP + cells displayed spontaneous (nonevoked) synaptic currents (Fig. 6A), suggesting that they receive synaptic inputs.

\section{Discussion}

GABAergic interneuron precursors from the MGE have the remarkable ability to migrate, differentiate, and function after transplantation into postnatal neocortex (Wichterle et al., 1999; Cobos et al., 2005; Alvarez-Dolado et al., 2006; Fogarty et al., 2007; Wonders et al., 2008). These characteristics make them attractive candidates for use in cell-based therapy, either by their intrinsic activity or as a vector for drug delivery, but such therapy would require the use of large numbers of cells. To demonstrate that MGE-like interneurons can be generated from ESCs, we took 
advantage of the fact that most of these neurons express the transcription factor Lhx6 from around the time they exit the cell cycle, through the phases of migration and initial differentiation, and into adulthood. A mouse ES line containing the Lhx6-GFP BAC construct allows for both the FACS collection of MGE-like interneuron precursors (Fig. 1), and the identification of these cells months after transplantation into the postnatal neocortex (Figs. 2-5).

Despite the relatively low percentage of GFP + cells generated $(\sim 2 \%)$ (Fig. 1), FACS collection results in hundreds of thousands of GFP + cells per experiment being generated for transplantation studies. Comparison of the results of these transplantations with similar neocortical transplantations of MGE-derived progenitors suggests that the Lhx6::GFP line can be used to generate GFP + cells with a differentiation potential remarkably similar to MGE progenitors (Figs. 2-6) (Cobos et al., 2005; Alvarez-Dolado et al., 2006; Flames et al., 2007; Wonders et al., 2008). Like MGE transplants into neonatal neocortex, Lhx6::GFP cells migrate extensively during the first week after transplantation (Fig. 4). However, detection of GFP + cells drops from $\sim 10 \%$ at 1 week to $2 \%$ at 1 month, whereas MGE transplant survival into neonatal cortex ranges from 10 to $20 \%$ after several weeks. There are a number of potential sources for this discrepancy. First, MGE transplants contain many $\mathrm{Nkx} 2.1+$ mitotic progenitors of the $\mathrm{VZ}$ and SVZ, whereas in this paper transplanted cells are exclusively at the Lhx6+ stage more characteristic of a subpopulation of MGE mantle zone cells. Second, the FACS sorting itself could influence long-term survivability. Third, MGE transplant experiments have mainly involved host and donor animals of the same strain, whereas in this paper the ES-line cells derive from the inbred 129J strain, while the host animals were of the highly outbred CD1 line, raising the possibility of substantial immune response. Finally, we have not ruled out the possibility of silencing of the GFP reporter in many cells. It has recently been reported that cholinergic striatal interneurons briefly express Lhx6 at the progenitor or immediately postmitotic stage (Fragkouli et al., 2009). This population is quite small relative to the GABAergic interneuron subpopulations of the striatum and cortex that maintain Lhx6 expression, but could be overrepresented in the conditions produced by the protocol used. Future studies will be needed to compare these results to those from transplantations of Lhx6::GFP + cells tagged also with a constitutively expressed marker, to Lhx6::GFP + cells generated by alternative protocols, to transplantations of FACS sorted, MGE cells from Lhx6-GFPexpressing transgenic mice, and to transplantations into immunesuppressed and 129J strain hosts.

Regardless of the survival issue, transplantations of Lhx6::GFP cells result in the successful grafting of large numbers of cells that can be studied after their maturation in vivo. Transplanted cells tend to have general morphologies of Lhx6-expressing cortical interneurons, including multipolar dendritic arborizations and aspiny dendrites (Figs. 5, 6). As occurs for Lhx6+ cells in vivo, the majority of transplanted Lhx6::GFP cells express either SST or PV (Fig. 5). Importantly, colabeling reveals that markers that are coexpressed with these subgroups, such as KV3.1 with the PV+ subgroup and NPY with some of the SST-expressing cells, are appropriately expressed in the transplanted ES-derived interneurons (Fig. 5). In addition, whole-cell patch-clamp recordings during the third and fourth postnatal weeks reveals neuronal characteristics, the presence of synaptic inputs, and spiking characteristics typical of Lhx6-expressing interneuron subgroups (Fig. 6). A recent study found that ES-derived neural precursor cells (ES-NPCs) were capable of forming distinct, subcortical projections to distant axonal targets based on where the ES-NPCs were transplanted in the cortex, suggesting that defined progenitors retain the intrinsic capacity to form connections within the CNS (Ideguchi et al., 2010). While further studies will be needed to fully describe the multiple types of interneurons generated in these studies at the important levels of axon targeting and downstream influences on single neuron, network activity, and animal behavior, the lines of evidence presented above strongly support the notion that the Lhx6::GFP ES line can be differentiated into interneuron-like cells.

The bias for Sst + interneurons over those that express PV was in some respects unexpected, since there are approximately two times more $\mathrm{PV}+$ than Sst + interneurons in mouse cortex (Tamamaki et al., 2003). The timing of FACS collection would not be expected to affect this ratio, as no overall differences in the birthdate of Sst + versus PV + subgroups are found within a given cortical layer (Wonders et al., 2008). However, based both on transplantation studies and genetic fate mapping, recent evidence suggests that within the MGE there is a partial bias for the generation of PV-expressing interneuron subgroups in the more ventral region of the MGE, while Sst-expressing subgroups are preferentially produced from progenitors located in the dorsal MGE (Flames et al., 2007; Fogarty et al., 2007; Wonders et al., 2008; Xu et al., 2008). This result suggests that the ventral telencephalic differentiation protocol we are using is preferentially directing the $\mathrm{Nkx} 2.1+$ progenitors toward dorsal MGE-like fates. As evidence suggests that the dorsal MGE domain is generated and maintained by high levels of Shh signaling compared with more ventral MGE regions (Xu et al., 2005; Wonders et al., 2008), and this higher level of Shh signaling contributes to the specification of Sst over PV interneuron subgroup fate (Xu et al., 2010), future experiments will examine whether alterations in timing or concentration of Shh results in Lhx6::GFP + cells characteristic of ventral MGE-derived interneuron types.

In sum, the evidence presented above is to our knowledge the first example of the use of reporter-modified ESCs for the prospective isolation of cells with potential for developing into ventral telencephalic subpopulations following transplantation and long-term survival in vivo. An important feature of Lhx6 + cells is their ability to retain migratory capacity and neuronal commitment after transplantation into the postnatal brain without forming tumors. These studies will enable the routine use of these cells for gene discovery and studies on interneuron development and function. Access to unlimited numbers of cells exhibiting these unique properties suggests considerable potential for the development of cell-based therapies in focal epilepsy and other forebrain disorders. In addition, the prospective isolation of ESC-derived neuron types at early stages of fate commitment represents a powerful paradigm that should be applicable for generating other types of forebrain neurons.

\section{References}

Alvarez-Dolado M, Calcagnotto ME, Karkar KM, Southwell DG, Jones-Davis DM, Estrada RC, Rubenstein JL, Alvarez-Buylla A, Baraban SC (2006) Cortical inhibition modified by embryonic neural precursors grafted into the postnatal brain. J Neurosci 26:7380-7389.

Anderson SA, Marín O, Horn C, Jennings K, Rubenstein JL (2001) Distinct cortical migrations from the medial and lateral ganglionic eminences. Development 128:353-363.

Ascoli GA, Alonso-Nanclares L, Anderson SA, Barrionuevo G, BenavidesPiccione R, Burkhalter A, Buzsáki G, Cauli B, Defelipe J, Fairén A, Feldmeyer D, Fishell G, Fregnac Y, Freund TF, Gardner D, Gardner EP, Goldberg JH, Helmstaedter M, Hestrin S, Karube F, et al. (2008) Petilla terminology: nomenclature of features of GABAergic interneurons of the cerebral cortex. Nat Rev Neurosci 9:557-568. 
Butt SJ, Fuccillo M, Nery S, Noctor S, Kriegstein A, Corbin JG, Fishell G (2005) The temporal and spatial origins of cortical interneurons predict their physiological subtype. Neuron 48:591-604.

Butt SJ, Sousa VH, Fuccillo MV, Hjerling-Leffler J, Miyoshi G, Kimura S, Fishell G (2008) The requirement of Nkx2-1 in the temporal specification of cortical interneuron subtypes. Neuron 59:722-732.

Cobos I, Calcagnotto ME, Vilaythong AJ, Thwin MT, Noebels JL, Baraban SC, Rubenstein JL (2005) Mice lacking Dlx1 show subtype-specific loss of interneurons, reduced inhibition and epilepsy. Nat Neurosci 8:10591068.

Du T, Xu Q, Ocbina PJ, Anderson SA (2008) NKX2.1 specifies cortical interneuron fate by activating Lhx6. Development 135:1559-1567.

Eiraku M, Watanabe K, Matsuo-Takasaki M, Kawada M, Yonemura S, Matsumura M, Wataya T, Nishiyama A, Muguruma K, Sasai Y (2008) Self-organized formation of polarized cortical tissues from ESCs and its active manipulation by extrinsic signals. Cell Stem Cell 3:519-532.

Flames N, Pla R, Gelman DM, Rubenstein JL, Puelles L, Marín O (2007) Delineation of multiple subpallial progenitor domains by the combinatorial expression of transcriptional codes. J Neurosci 27:9682-9695.

Fogarty M, Grist M, Gelman D, Marín O, Pachnis V, Kessaris N (2007) Spatial genetic patterning of the embryonic neuroepithelium generates GABAergic interneuron diversity in the adult cortex. J Neurosci 27:10935-10946.

Fragkouli A, van Wijk NV, Lopes R, Kessaris N, Pachnis V (2009) LIM homeodomain transcription factor-dependent specification of bipotential MGE progenitors into cholinergic and GABAergic striatal interneurons. Development 136:3841-3851.

Gaspard N, Bouschet T, Hourez R, Dimidschstein J, Naeije G, van den Ameele J, Espuny-Camacho I, Herpoel A, Passante L, Schiffmann SN, Gaillard A, Vanderhaeghen P (2008) An intrinsic mechanism of corticogenesis from embryonic stem cells. Nature 455:351-357.

Gonchar Y, Burkhalter A (1997) Three distinct families of GABAergic neurons in rat visual cortex. Cereb Cortex 7:347-358.

Gong S, Zheng C, Doughty ML, Losos K, Didkovsky N, Schambra UB, Nowak NJ, Joyner A, Leblanc G, Hatten ME, Heintz N (2003) A gene expression atlas of the central nervous system based on bacterial artificial chromosomes. Nature 425:917-925.

Grigoriou M, Tucker AS, Sharpe PT, Pachnis V (1998) Expression and regulation of Lhx6 and Lhx7, a novel subfamily of LIM homeodomain encoding genes, suggests a role in mammalian head development. Development 125:2063-2074.

Ideguchi M, Palmer TD, Recht LD, Weimann JM (2010) Murine embryonic stem cell-derived pyramidal neurons integrate into the cerebral cortex and appropriately project axons to subcortical targets. J Neurosci 30:894-904.

Kawaguchi Y, Kubota Y (1996) Physiological and morphological identification of somatostatin- or vasoactive intestinal polypeptide-containing cells among GABAergic cell subtypes in rat frontal cortex. J Neurosci 16:2701-2715.

Kubota Y, Kawaguchi Y (1994) Three classes of GABAergic interneurons in neocortex and neostriatum. Jpn J Physiol 44 [Suppl 2]:S145-S148.

Lavdas AA, Grigoriou M, Pachnis V, Parnavelas JG (1999) The medial ganglionic eminence gives rise to a population of early neurons in the developing cerebral cortex. J Neurosci 19:7881-7888.

Lee SH, Lumelsky N, Studer L, Auerbach JM, McKay RD (2000) Efficient generation of midbrain and hindbrain neurons from mouse embryonic stem cells. Nat Biotechnol 18:675-679.

Levitt P, Eagleson KL, Powell EM (2004) Regulation of neocortical inter- neuron development and the implications for neurodevelopmental disorders. Trends Neurosci 27:400-406.

Liodis P, Denaxa M, Grigoriou M, Akufo-Addo C, Yanagawa Y, Pachnis V (2007) Lhx6 activity is required for the normal migration and specification of cortical interneuron subtypes. J Neurosci 27:3078-3089.

Markram H, Toledo-Rodriguez M, Wang Y, Gupta A, Silberberg G, Wu C (2004) Interneurons of the neocortical inhibitory system. Nat Rev Neurosci 5:793-807.

Miyoshi G, Butt SJ, Takebayashi H, Fishell G (2007) Physiologically distinct temporal cohorts of cortical interneurons arise from telencephalic Olig2expressing precursors. J Neurosci 27:7786-7798.

Porteus MH, Bulfone A, Liu JK, Puelles L, Lo LC, Rubenstein JLR (1994) DLX-2, MASH-1, and MAP-2 expression and bromodeoxyuridine incorporation define molecularly distinct cell populations in the embryonic mouse forebrain. J Neurosci 14:6370-6383.

Tabar V, Tomishima M, Panagiotakos G, Wakayama S, Menon J, Chan B, Mizutani E, Al-Shamy G, Ohta H, Wakayama T, Studer L (2008) Therapeutic cloning in individual parkinsonian mice. Nat Med 14:379-381.

Tamamaki N, Yanagawa Y, Tomioka R, Miyazaki J, Obata K, Kaneko T (2003) Green fluorescent protein expression and colocalization with calretinin, parvalbumin, and somatostatin in the GAD67-GFP knock-in mouse. J Comp Neurol 467:60-79.

Tomishima MJ, Hadjantonakis AK, Gong S, Studer L (2007) Production of green fluorescent protein transgenic embryonic stem cells using the GENSAT bacterial artificial chromosome library. Stem Cells 25:39-45.

Watanabe K, Kamiya D, Nishiyama A, Katayama T, Nozaki S, Kawasaki H, Watanabe Y, Mizuseki K, Sasai Y (2005) Directed differentiation of telencephalic precursors from embryonic stem cells. Nat Neurosci $8: 288-296$.

Wichterle H, Garcia-Verdugo JM, Herrera DG, Alvarez-Buylla A (1999) Young neurons from medial ganglionic eminence disperse in adult and embryonic brain. Nat Neurosci 2:461-466.

Wichterle H, Lieberam I, Porter JA, Jessell TM (2002) Directed differentiation of embryonic stem cells into motor neurons. Cell 110:385-397.

Wichterle H, Alvarez-Dolado M, Erskine L, Alvarez-Buylla A (2003) Permissive corridor and diffusible gradients direct medial ganglionic eminence cell migration to the neocortex. Proc Natl Acad Sci U S A 100: $727-732$.

Wonders CP, Taylor L, Welagen J, Mbata IC, Xiang JZ, Anderson SA (2008) A spatial bias for the origins of interneuron subgroups within the medial ganglionic eminence. Dev Biol 314:127-136.

Xu Q, Cobos I, De La Cruz E, Rubenstein JL, Anderson SA (2004) Origins of cortical interneuron subtypes. J Neurosci 24:2612-2622.

Xu Q, Wonders CP, Anderson SA (2005) Sonic hedgehog maintains the identity of cortical interneuron progenitors in the ventral telencephalon. Development 132:4987-4998.

Xu Q, Tam M, Anderson SA (2008) Fate mapping Nkx2.1-lineage cells in the mouse telencephalon. J Comp Neurol 506:16-29.

Xu Q, Guo L, Moore H, Waclaw RR, Campbell K, Anderson SA (2010) Sonic hedgehog signaling confers ventral telencephalic progenitors with distinct cortical interneuron fates. Neuron 65:328-340.

Xu X, Roby KD, Callaway EM (2006) Mouse cortical inhibitory neuron type that coexpresses somatostatin and calretinin. J Comp Neurol 499:144160.

Zhao Y, Flandin P, Long JE, Cuesta MD, Westphal H, Rubenstein JL (2008) Distinct molecular pathways for development of telencephalic interneuron subtypes revealed through analysis of Lhx6 mutants. J Comp Neurol 510:79-99. 